Bull. Korean Math. Soc. 50 (2013), No. 5, pp. 1501-1511

http://dx.doi.org/10.4134/BKMS.2013.50.5.1501

\title{
Extensions of linearly McCoy rings
}

\author{
JiAn Cui AND JiAnlong Chen
}

\begin{abstract}
A ring $R$ is called linearly McCoy if whenever linear polynomials $f(x), g(x) \in R[x] \backslash\{0\}$ satisfy $f(x) g(x)=0$, there exist nonzero elements $r, s \in R$ such that $f(x) r=s g(x)=0$. In this paper, extension properties of linearly McCoy rings are investigated. We prove that the polynomial ring over a linearly McCoy ring need not be linearly McCoy. It is shown that if there exists the classical right quotient $\operatorname{ring} Q$ of a ring $R$, then $R$ is right linearly McCoy if and only if so is $Q$. Other basic extensions are also considered.
\end{abstract}

\section{Introduction}

All rings are associative with unity. For a ring $R$, the polynomial ring over $R$ is denoted by $R[x]$ with $x$ its indeterminate, and $E_{i j}$ stands for the usual matrix unit (i.e., with 1 at $(i, j)$-entry and 0 elsewhere).

McCoy proved in 1942 [14] that if two polynomials annihilate each other over a commutative ring, then each polynomial has a nonzero annihilator in the base ring. Rege and Chhawchharia [16] and Nielsen [15] independently introduced the notion of a McCoy ring. A ring $R$ is right McCoy if the equation $f(x) g(x)=0$ with $f(x), g(x) \in R[x] \backslash\{0\}$ implies there exists a nonzero $r \in R$ such that $f(x) r=0$; left McCoy rings are defined similarly. A ring $R$ is called McCoy if it is both right and left McCoy. The class of McCoy rings contains the class of Armendariz rings (These rings are defined through the condition 'whenever polynomials $f(x)=\sum_{i=0}^{m} a_{i} x^{i}, g(x)=\sum_{j=0}^{n} b_{j} x^{j} \in R[x]$ satisfy $f(x) g(x)=0$, then $a_{i} b_{j}=0$ for every $i$ and $\left.j[16]^{\prime}\right)$. A ring $R$ is semi-commutative provided $a b=0$ implies $a R b=0$ for $a, b \in R$. In [7] it was claimed that all semi-commutative rings were McCoy. However, Hirano's claim assumed that $R[x]$ is semi-commutative if $R$ is semi-commutative, and this was shown to be false in [8]. In 2006, Nielsen [15] gave an example of semi-commutative ring which is not right McCoy. The concept of a linearly McCoy ring, which properly generalizes McCoy rings and semi-commutative rings, was introduced by Camillo and Nielsen [4] in 2008. Recall that a ring $R$

Received April 8, 2012.

2010 Mathematics Subject Classification. Primary 16U80; Secondary 16S99.

Key words and phrases. polynomial ring, linearly McCoy ring, matrix ring, semicommutative ring, McCoy ring. 
is called (right or left) linearly McCoy if the McCoy condition holds for nonzero linear polynomials $f(x)=a_{0}+a_{1} x, g(x)=b_{0}+b_{1} x \in R[x]$. Related results on McCoy conditions can be found in $[4,5,10,12,15,16,17]$, etc. Recently, the McCoy and the Armendariz conditions were extended to their module versions (see [3, 6]). Due to Lee and Wong [11], a $\operatorname{ring} R$ is called weak Armendariz (also called linearly Armendariz in literature) if for given $f(x)=a_{0}+a_{1} x$ and $g(x)=b_{0}+b_{1} x \in R[x], f(x) g(x)=0$ implies that $a_{i} b_{j}=0$ for each $i, j$. Weak Armendariz rings are clearly linearly McCoy; the falsity of the converse can be deduced from [9, Example 1.2(4)].

The polynomial extension property of rings plays an important role in ring theory. For rings that admit Armendariz or McCoy condition, it was proved in [1] (resp., [12]) that a ring $R$ is Armendariz (resp., McCoy) if and only if $R[x]$ is Armendariz (resp., McCoy). But it is still an open question of whether the polynomial ring over a weak Armendariz ring is weak Armendariz (see [9]). In this paper, we show that the polynomial ring over a semi-commutative ring is not linearly McCoy. It is proved that if $R$ is a linearly McCoy ring, then $R[x] /\left(x^{n}\right)$ is linearly McCoy for any integer $n \geq 1$; and if there exists a classical right quotient ring $Q$ of a ring $R$, then $R$ is right linearly McCoy if and only if $Q$ is right linearly McCoy. Some other basic extensions of linearly McCoy rings are also considered.

\section{Polynomial rings}

In this section, we investigate the polynomial ring over a linearly McCoy ring. We first recall a fact in [4].

Lemma 2.1. All semi-commutative rings are linearly McCoy.

Anderson and Camillo [1] proved that a ring $R$ is Armendariz if and only if $R[x]$ is Armendariz, and Lei et al. [12] showed the same property holds for McCoy rings. It is natural to consider whether polynomial rings over linearly McCoy rings are still linearly McCoy. Motivated by results in [15], we have the following result.

Theorem 2.2. There exists a semi-commutative ring over which the polynomial ring is not linearly McCoy.

Proof. Let $K=\mathbb{F}_{2}\left\langle a_{0}, a_{1}, a_{2}, a_{3}, a_{4}, b_{0}, b_{1}, b_{2}, b_{3}\right\rangle$ be the free associative algebra (with 1 ) over $\mathbb{F}_{2}$ generated by nine indeterminates (as labeled above). Let $I$ be the ideal generated by the following relations:

$$
\begin{aligned}
& \left\langle a_{0} b_{0}, a_{0} b_{1}+a_{1} b_{0}, a_{0} b_{2}+a_{1} b_{1}+a_{2} b_{0}, a_{1} b_{2}+a_{2} b_{1}+a_{3} b_{0}, a_{2} b_{2}+a_{3} b_{1}, a_{3} b_{2},\right. \\
& a_{0} b_{3}+a_{4} b_{0}, a_{1} b_{3}+a_{4} b_{1}, a_{2} b_{3}+a_{4} b_{2}, a_{3} b_{3}, a_{4} b_{3}, a_{0} a_{j}(0 \leq j \leq 4), \\
& a_{3} a_{j}(0 \leq j \leq 4), a_{4} a_{j}(0 \leq j \leq 4), a_{1} a_{j}+a_{2} a_{j}(0 \leq j \leq 4), \\
& \left.b_{i} b_{j}(0 \leq i, j \leq 3), b_{i} a_{j}(0 \leq i \leq 3,0 \leq j \leq 4)\right\rangle .
\end{aligned}
$$


Let $R=K / I$. Set $F(y)=a_{0}+a_{1} x+a_{2} x^{2}+a_{3} x^{3}+a_{4} y, G(y)=b_{0}+b_{1} x+$ $b_{2} x^{2}+b_{3} y$. Note that the first and the second rows of relations in $I$ guarantee $F(y) G(y)=0$ in $R[x][y]$.

The degree of each nonzero monomial $\alpha \in R$ is defined as the number of indeterminates in $\alpha$, denote it by $\operatorname{deg}(\alpha)$; the degree of each element $\gamma=$ $\sum_{i} r_{i} \in R$ is defined as $\operatorname{deg}(\gamma)=\max \left\{\operatorname{deg}\left(r_{i}\right): i \in \mathbb{N}\right\}$, where $r_{i}$ is the part of degree $i$ in $\gamma$.

Let $H_{n}$ be the set of all linear combinations of monomials of degree $n$ over $\mathbb{F}_{2}$. Notice that $H_{n}$ is finite for any $n$ and the ideal $I$ of $R$ is homogeneous (i.e., if $\sum_{i=1}^{s} r_{i} \in I$ with $r_{i} \in H_{i}$, then $\left.r_{i} \in I\right)$. The proof will be divided into the following four claims.

Claim 1. Let $\gamma \in R$. Then

$$
\begin{aligned}
\gamma= & f_{0}+f_{1}\left(a_{2}\right) a_{1}+f_{2}\left(a_{2}\right) a_{2}+f_{3}\left(a_{2}\right) a_{3}+f_{4}\left(a_{2}\right) a_{4}+g\left(a_{2}\right) a_{0} \\
& +\left[r_{0}+r_{1}\left(a_{2}\right) a_{1}+r_{2}\left(a_{2}\right) a_{2}+r_{3}\left(a_{2}\right) a_{3}+r_{4}\left(a_{2}\right) a_{4}\right] b_{0} \\
& +\left[s_{0}+s_{1}\left(a_{2}\right) a_{1}+s_{2}\left(a_{2}\right) a_{2}+s_{3}\left(a_{2}\right) a_{3}+s_{4}\left(a_{2}\right) a_{4}\right] b_{1} \\
& +\left[t_{0}+t\left(a_{2}\right) a_{4}\right] b_{2}+h_{0} b_{3}
\end{aligned}
$$

with $f_{0}, r_{0}, s_{0}, t_{0}, h_{0} \in \mathbb{F}_{2}$ and $f_{i}, g, r_{j}, s_{k}, t \in \mathbb{F}_{2}[x](1 \leq i, j, k \leq 4)$, and the expression is unique.

Proof of Claim 1. We directly adopt the diamond lemma (see [2]), one may reduce any given monomial through the relations specified in the definition of $I$ as follows.

Firstly, check whether the monomial we plan to reduce has any occurrence of $a_{0} b_{0}, a_{3} b_{2}, a_{3} b_{3}, a_{4} b_{3}, a_{0} a_{j}, a_{3} a_{j}, a_{4} a_{j}, b_{i} b_{j}, b_{i} a_{j}$. If so, then the monomial is zero. If not, repeatedly replace all occurrences of $a_{0} b_{1}, a_{0} b_{2}, a_{1} b_{2}, a_{2} b_{2}, a_{0} b_{3}$, $a_{1} b_{3}, a_{2} b_{3}, a_{1} a_{j}$ with $a_{1} b_{0}, a_{1} b_{1}+a_{2} b_{0}, a_{2} b_{1}+a_{3} b_{0}, a_{3} b_{1}, a_{4} b_{0}, a_{4} b_{1}, a_{4} b_{2}, a_{2} a_{j}$, respectively. Then the resulting monomial will be in reduced form, and any element of $R$ is just a sum of monomials. Hence, each element of $R$ can be written uniquely as the form above.

Claim 2. The $\operatorname{ring} R$ is semi-commutative.

Proof of Claim 2. Let $\gamma, \gamma^{\prime} \in R$ with $\gamma \gamma^{\prime}=0$, where $\gamma, \gamma^{\prime}$ are written in the form of Claim 1 . We write $f_{1}$ for $f_{1}\left(a_{2}\right)$, and do the same for other polynomials in the variable $a_{2}$. Then $\gamma=f_{0}+f_{1} a_{1}+f_{2} a_{2}+\cdots+h_{0} b_{3}, \gamma^{\prime}=f_{0}^{\prime}+f_{1}^{\prime} a_{1}+$ $f_{2}^{\prime} a_{2}+\cdots+h_{0}^{\prime} b_{3}$. Throughout we use the fact that $I$ is a homogeneous ideal, so the sum of all monomials of any given degree in $\gamma \gamma^{\prime}$ is zero.

For all $r \in R$, we prove that $\gamma r \gamma^{\prime}=0$. Clearly, it is true if either $\gamma$ or $\gamma^{\prime}$ is zero. So we may assume that $\gamma, \gamma^{\prime}$ are nonzero in $R . \gamma \gamma^{\prime}=0$ implies that $f_{0} f_{0}^{\prime}=0$. So $f_{0}=0$ or $f_{0}^{\prime}=0$. Suppose that $f_{0}=0$. Let $\lambda \neq 0$ be the sum of the (nonzero) terms of $\gamma$ with lowest degree. Since $I$ is homogeneous, $\lambda f_{0}^{\prime}=0$, and so $f_{0}^{\prime}=0$. Similarly, if $f_{0}^{\prime}=0$ we obtain $f_{0}=0$. Thus, $f_{0}=f_{0}^{\prime}=0$. 
Suppose that $\operatorname{deg}(r)=1$. Notice that $b_{i} \gamma^{\prime}=0$ for $0 \leq i \leq 3$ since $f_{0}^{\prime}=0$. Therefore $\gamma b_{i} \gamma^{\prime}=0$. So it suffices to check $\gamma a_{j} \gamma^{\prime}=0$ for $0 \leq j \leq 4$. By Claim 1 , we get $\gamma a_{j}=\left(f_{1}+f_{2}\right) a_{2} a_{j}$. So if $f_{1}=f_{2}$, then we have $\gamma a_{j} \gamma^{\prime}=0$. In what follows, assume that $f_{1} \neq f_{2}$. We show below that this contradicts the assumption $\gamma^{\prime} \neq 0$.

Computing the reduced form of $\gamma \gamma^{\prime}$ yields

$$
\begin{aligned}
0= & \gamma \gamma^{\prime} \\
= & \left(f_{1}+f_{2}\right) a_{2}\left(f_{1}^{\prime} a_{1}+f_{2}^{\prime} a_{2}+f_{3}^{\prime} a_{3}+f_{4}^{\prime} a_{4}+g^{\prime} a_{0}\right) \\
& +\left(r_{0}^{\prime} f_{1}+s_{0}^{\prime} g+\left(f_{1}+f_{2}\right) a_{2} r_{1}^{\prime}\right) a_{1} b_{0}+\left(r_{0}^{\prime} f_{2}+t_{0}^{\prime} g+\left(f_{1}+f_{2}\right) a_{2} r_{2}^{\prime}\right) a_{2} b_{0} \\
& +\left(r_{0}^{\prime} f_{3}+t_{0}^{\prime} f_{1}+\left(f_{1}+f_{2}\right) a_{2} r_{3}^{\prime}\right) a_{3} b_{0}+\left(r_{0}^{\prime} f_{4}+h_{0}^{\prime} g+\left(f_{1}+f_{2}\right) a_{2} r_{4}^{\prime}\right) a_{4} b_{0} \\
& +\left(s_{0}^{\prime} f_{1}+t_{0}^{\prime} g+\left(f_{1}+f_{2}\right) a_{2} s_{1}^{\prime}\right) a_{1} b_{1}+\left(s_{0}^{\prime} f_{2}+t_{0}^{\prime} f_{1}+\left(f_{1}+f_{2}\right) a_{2} s_{2}^{\prime}\right) a_{2} b_{1} \\
& +\left(s_{0}^{\prime} f_{3}+t_{0}^{\prime} f_{2}+\left(f_{1}+f_{2}\right) a_{2} s_{3}^{\prime}\right) a_{3} b_{1}+\left(s_{0}^{\prime} f_{4}+h_{0}^{\prime} f_{1}+\left(f_{1}+f_{2}\right) a_{2} s_{4}^{\prime}\right) a_{4} b_{1} \\
& +\left(t_{0}^{\prime} f_{4}+h_{0}^{\prime} f_{2}+\left(f_{1}+f_{2}\right) a_{2} t^{\prime}\right) a_{4} b_{2} .
\end{aligned}
$$

For ease of notation, we denote the coefficient of $a_{k} b_{l}$ in $\gamma \gamma^{\prime}$ by $\circledast_{a_{k}} b_{l}$. That is

$$
\begin{aligned}
\circledast_{a_{1} b_{0}} & =r_{0}^{\prime} f_{1}+s_{0}^{\prime} g+\left(f_{1}+f_{2}\right) a_{2} r_{1}^{\prime}, \\
\circledast_{a_{2} b_{0}} & =r_{0}^{\prime} f_{2}+t_{0}^{\prime} g+\left(f_{1}+f_{2}\right) a_{2} r_{2}^{\prime}, \\
& \cdots \\
\circledast_{a_{4} b_{2}} & =t_{0}^{\prime} f_{4}+h_{0}^{\prime} f_{2}+\left(f_{1}+f_{2}\right) a_{2} t^{\prime} .
\end{aligned}
$$

Since $f_{1}+f_{2} \neq 0$, it follows that $f_{1}^{\prime}=f_{2}^{\prime}=f_{3}^{\prime}=f_{4}^{\prime}=g^{\prime}=0$. From the last five lines of $\gamma \gamma^{\prime}$ we obtain

$$
\circledast a_{i} b_{0}=\circledast a_{a_{i} b_{1}}=\circledast a_{a_{4} b_{2}}=0, \quad i=1, \ldots, 4 .
$$

Assume that $s_{0}^{\prime}=1$. If $t_{0}^{\prime}=1$, then Eq. $\circledast_{a_{2} b_{1}}$ implies $\operatorname{deg}\left(\left(f_{1}+f_{2}\right) a_{2}\right) \leq$ $\operatorname{deg}\left(f_{1}+f_{2}\right)$, which is impossible since $f_{1} \neq f_{2}$. So $t_{0}^{\prime}=0$. But then adding Eqs. $\circledast a_{1} b_{1}$ and $\circledast a_{2} b_{1}$ causes the same contradiction.

Thus we have $s_{0}^{\prime}=0$. If $t_{0}^{\prime}=1$, then adding Eqs. $\circledast_{a_{2} b_{1}}$ and $\circledast_{a_{3} b_{1}}$ reaches the same contradiction as above. Therefore, $t_{0}^{\prime}=0$.

Suppose that $r_{0}^{\prime}=1$ and $h_{0}^{\prime}=1$, then adding Eqs. $\circledast a_{1} b_{0}$ and $\circledast a_{2} b_{0}$, Eqs. $\circledast a_{4} b_{1}$ and $\circledast a_{4} b_{2}$, respectively. We also obtain the preceding contradiction. So $r_{0}^{\prime}=0$ and $h_{0}^{\prime}=0$. Because $f_{1} \neq f_{2}$, we have $r_{i}^{\prime}=s_{j}^{\prime}=t^{\prime}=0$, where $1 \leq i, j \leq 4$. Hence $\gamma^{\prime}=0$, contradicting our previous assumption that $\gamma^{\prime} \neq 0$.

This shows that $\gamma r \gamma^{\prime}=0$ if $\operatorname{deg}(r)=1$. Repeating the above argument replacing $\gamma$ by $\gamma r, \gamma r \gamma^{\prime}=0$ also holds when $r$ is a monomial of any positive degree. Clearly, if $r=1$ then $\gamma r \gamma^{\prime}=0$. Since any element of $R$ is just a sum of monomials, putting this all together yields $\gamma r \gamma^{\prime}=0$ for all $r \in R$. Therefore, $R$ is a semi-commutative ring.

Due to Claim 1, one may check that $F(y), G(y) \neq 0$ in $R[x][y]$.

Claim 3. The polynomial ring $R[x]$ is not right linearly McCoy. 
Proof of Claim 3. We conclude that $r_{R[x]}\left(a_{4}\right)=\left\{\sum_{i} k_{i} b_{3} x^{i}: k_{i} \in \mathbb{F}_{2}, i \in \mathbb{N}\right\}+$ $\sum_{j=0}^{4} a_{j} R[x]$. Obviously, by the construction of the ideal $I$, each polynomial of the right side set in the equation above annihilates $a_{4}$ on the right. Meanwhile, for any $p(x) \in r_{R[x]}\left(a_{4}\right)$, write $p(x)=\gamma_{0}+\gamma_{1} x+\gamma_{2} x^{2}+\cdots+\gamma_{n} x^{n}$ with $\gamma_{i} \in R$ written in the form as Claim 1. So we let $\gamma_{i}=f_{0}^{(i)}+f_{1}^{(i)} a_{1}+\cdots+h_{0}^{(i)} b_{3}, 0 \leq$ $i \leq n$. Since $a_{4} \gamma_{i}=0$, we have $f_{0}^{(i)}=r_{0}^{(i)}=s_{0}^{(i)}=t_{0}^{(i)}=0$. Thus $\gamma_{i} \in\left\{k b_{3}\right.$ : $\left.k \in \mathbb{F}_{2}\right\}+\sum_{j=0}^{4} a_{j} R$, i.e., $p(x) \in\left\{\sum_{i} k_{i} b_{3} x^{i}: k_{i} \in \mathbb{F}_{2}, i \in \mathbb{N}\right\}+\sum_{j=0}^{4} a_{j} R[x]$.

For any $p(x) \in R[x]$, if $F(y) p(x)=0$, then $p(x) \in r_{R[x]}\left(a_{4}\right)$ since $a_{4} p(x)=$ 0 . So let $p(x)=\left(k_{0} b_{3}+k_{0}^{\prime} p_{0}\right)+\left(k_{1} b_{3}+k_{1}^{\prime} p_{1}\right) x+\left(k_{2} b_{3}+k_{2}^{\prime} p_{2}\right) x^{2}+\cdots+\left(k_{n} b_{3}+\right.$ $\left.k_{n}^{\prime} p_{n}\right) x^{n}$, where $k_{i}, k_{i}^{\prime} \in \mathbb{F}_{2}, p_{l} \in \sum_{j=0}^{4} a_{j} R, 0 \leq l \leq n$, and each $p_{l}$ is written uniquely as Claim 1. If some $p_{l_{0}}$ does not occur in the coefficients of $p(x)$ (i.e., $p_{l_{0}}=0$ ), then let $k_{l_{0}}^{\prime}=0$.

From the equation $F(y) p(x)=0$, we also get $\left(a_{0}+a_{1} x+a_{2} x^{2}+a_{3} x^{3}\right) p(x)=0$. This implies the following system of equations:

(0) $a_{0}\left(k_{0} b_{3}+k_{0}^{\prime} p_{0}\right)=0$,

(1) $a_{0}\left(k_{1} b_{3}+k_{1}^{\prime} p_{1}\right)+a_{1}\left(k_{0} b_{3}+k_{0}^{\prime} p_{0}\right)=0$,

(2) $a_{0}\left(k_{2} b_{3}+k_{2}^{\prime} p_{2}\right)+a_{1}\left(k_{1} b_{3}+k_{1}^{\prime} p_{1}\right)+a_{2}\left(k_{0} b_{3}+k_{0}^{\prime} p_{0}\right)=0$,

(3) $a_{0}\left(k_{3} b_{3}+k_{3}^{\prime} p_{3}\right)+a_{1}\left(k_{2} b_{3}+k_{2}^{\prime} p_{2}\right)+a_{2}\left(k_{1} b_{3}+k_{1}^{\prime} p_{1}\right)+a_{3}\left(k_{0} b_{3}+k_{0}^{\prime} p_{0}\right)=0$,

$(n+1) a_{1}\left(k_{n} b_{3}+k_{n}^{\prime} p_{n}\right)+a_{2}\left(k_{n-1} b_{3}+k_{n-1}^{\prime} p_{n-1}\right)+a_{3}\left(k_{n-2} b_{3}+k_{n-2}^{\prime} p_{n-2}\right)=0$.

Notice that $a_{0} p_{i}=0$ and $a_{k} p_{i}=a_{k} b_{3}=0$, where $k=3,4 ; i=0,1, \ldots, n$. So from Eq.(0) we have $k_{0}=0$, and Eq. (1) implies that $k_{0}^{\prime}=0, k_{1}=0$. Continuing this process, Eq. $(i+1)$ yields $k_{i}^{\prime}=k_{i+1}=0$ for $1 \leq i \leq n-1$, and we get $k_{n}^{\prime}=0$ from Eq. $(n+1)$. Thus, $p(x)=0$, which implies that $R[x]$ is not right linearly McCoy.

Claim 4. The ring $R$ is left McCoy (so $R[x]$ is left linearly McCoy).

Proof of Claim 4. For completeness of the proof, we adapt the method used in [15, Claim 8]. Let $\alpha(x), \beta(x) \in R[x] \backslash\{0\}$ satisfy $\alpha(x) \beta(x)=0$. Set $\alpha(x)=$ $\sum_{i=0}^{m} p_{i} x^{i}, \beta(x)=\sum_{i=0}^{n} q_{i} x^{i}$. If each $q_{i}$ has zero constant term, then $b_{0} q_{i}=$ 0 , whence $b_{0} \beta(x)=0$, and we are done. Next we assume that there exists some $q_{i}$ has a nonzero constant term. Let $l_{0}$ be the smallest index such that $q_{l_{0}}$ satisfies this property.

For each $p_{i} \neq 0$, let $p_{i}^{\prime}$ be the sum of nonzero terms of $p_{i}$ with smallest degree. And for $p_{i}=0$, put $p_{i}^{\prime}=0$. Also, let $k_{0}$ be the smallest index such that, among the members of $\left\{p_{0}^{\prime}, p_{1}^{\prime}, \ldots, p_{m}^{\prime}\right\} \backslash\{0\}$, we have $p_{k_{0}}^{\prime}$ with minimal degree, since $\alpha(x) \neq 0, k_{0}$ exists.

Notice that the degree $l_{0}+k_{0}$ part of $\alpha(x) \beta(x)=0$ we obtain

$$
\sum_{s, t: s+t=l_{0}+k_{0}} p_{s} q_{t}=0
$$


Since $I$ is a homogeneous ideal, each term of any fixed degree in Eq. $(*)$ must add to zero. From our choice of $k_{0}$ and $l_{0}$, the term of smallest degree in Eq. $(*)$ is $p_{k_{0}}^{\prime} \cdot 1=p_{k_{0}}^{\prime} \neq 0$, which comes from $p_{k_{0}} q_{l_{0}}$. So this causes a contradiction.

Hence $R$ is a left McCoy ring. In view of [12, Theorem 1], the polynomial $\operatorname{ring} R[x]$ is left linearly McCoy.

This completes the proof of Theorem 2.2.

By virtue of Theorem 2.2, a well-known result relating to semi-commutativity can be obtained (cf. [4, Example 8.6], [8, Example 2]).

Corollary 2.3. The polynomial rings over semi-commutative rings need not be semi-commutative.

Proposition 2.4. Let $R$ be a ring and $\Omega$ be a multiplicatively closed subset of $R$ consisting of central regular elements. Then $R$ is linearly McCoy if and only if $\Omega^{-1} R$ is linearly McCoy.

Proof. " $\Rightarrow$ ". Let $f(x)=\alpha+\beta x, g(x)=\alpha^{\prime}+\beta^{\prime} x$ be nonzero elements of $\Omega^{-1} R[x]$ such that $f(x) g(x)=0$, where $\alpha, \beta, \alpha^{\prime}, \beta^{\prime} \in \Omega^{-1} R$. Then there exist $u, v \in \Omega$ such that $\alpha=u^{-1} a, \beta=u^{-1} b, \alpha^{\prime}=v^{-1} c, \beta^{\prime}=v^{-1} d$. Since $\Omega$ is contained in the center of $R$, we have $f(x) g(x)=u^{-1}(a+b x) v^{-1}(c+d x)=$ $(u v)^{-1}(a+b x)(c+d x)=0$. Set $f_{1}(x)=a+b x, g_{1}(x)=c+d x$. Obviously, $f_{1}(x), g_{1}(x) \in R[x] \backslash\{0\}$ and $f_{1}(x) g_{1}(x)=0$. There exist nonzero $s, t \in R$ such that $f_{1}(x) s=t g_{1}(x)=0$ since $R$ is linearly McCoy. Then $f(x) \gamma=\delta g(x)=0$, where $\gamma=w^{-1} s, \delta=w^{-1} t$ and nonzero $w \in \Omega$. Therefore, $\Omega^{-1} R$ is linearly McCoy.

" $\Leftarrow "$. Suppose that $f(x)=a_{0}+a_{1} x$ and $g(x)=b_{0}+b_{1} x$ are nonzero elements of $R[x]$ with $f(x) g(x)=0$. Also, $f(x), g(x) \in \Omega^{-1} R[x] \backslash\{0\}$. Because $\Omega^{-1} R$ is linearly McCoy, there exist $\alpha=u^{-1} a, \beta=v^{-1} b \in \Omega^{-1} R \backslash\{0\}$ such that $f(x) \alpha=\beta g(x)=0$. It follows that $f(x) a=b g(x)=0$. Thus $R$ is linearly $\mathrm{McCoy}$.

The ring of Laurent polynomials in $x$, coefficients in a ring $R$, consisting of all formal sums $\sum_{i=k}^{n} m_{i} x^{i}$ with obvious addition and multiplication, where $m_{i} \in R$ and $k, n$ are (possibly negative) integers; denote it by $R\left[x ; x^{-1}\right]$.

Corollary 2.5. For a ring $R, R[x]$ is linearly $M c$ Coy if and only if $R\left[x ; x^{-1}\right]$ is linearly McCoy.

Proof. Let $\Omega=\left\{1, x, x^{2}, \ldots\right\}$. Then $\Omega$ is a multiplicatively closed subset of $R[x]$ consisting entirely of central regular elements. Since $R\left[x ; x^{-1}\right]=\Omega^{-1} R[x]$, by Proposition 2.4, we are done.

\section{Matrix rings and classical quotient rings}

In this section, we study the property "linearly McCoy" of some subring of the upper triangular matrix ring; the trivial extension of a linearly McCoy ring and its classical quotient ring are also investigated. 
Let $R$ be a ring. We consider the ring

$$
R_{n}=\left\{\left(\begin{array}{ccccc}
a & a_{12} & a_{13} & \ldots & a_{1 n} \\
0 & a & a_{23} & \ldots & a_{2 n} \\
0 & 0 & a & \ldots & a_{3 n} \\
\vdots & \vdots & \vdots & \ddots & \vdots \\
0 & 0 & 0 & \ldots & a
\end{array}\right): a, a_{k l} \in R\right\}
$$

where $n(\geq 1)$ is a positive integer.

Proposition 3.1. For any $n \geq 1$, a ring $R$ is linearly McCoy if and only if the ring $R_{n}$ is linearly McCoy.

Proof. " $\Rightarrow$ ". Let $H(x) \in R_{n}[x]$. Then $H(x)$ can be expressed as the form of a matrix, and the $(i, j)$-entry of $H(x)$ is denoted by $h_{i j}(x)=[H(x)]_{i, j}$. Clearly, $h_{i j}(x) \in R[x]$.

Suppose that $F(x), G(x)$ are nonzero linear polynomials of $R_{n}[x]$ with $F(x) G(x)=0$. We show that there exist $A, B \in R_{n} \backslash\{0\}$ such that $F(x) A=$ $B G(x)=0$. Now we proceed with the following cases.

Case 1. If $f_{11}(x) \neq 0, g_{11}(x) \neq 0$, then $f_{11}(x) g_{11}(x)=0$, where $f_{11}(x)=$ $[F(x)]_{1,1}, g_{11}(x)=[G(x)]_{1,1}$. Since $R$ is linearly McCoy, there exist $s, t \in$ $R \backslash\{0\}$ such that $f_{11}(x) s=t g_{11}(x)=0$. Put $A=s E_{1 n}, B=t E_{1 n}$. Then $F(x) A=B G(x)=0$.

Case 2. If $f_{11}(x) \neq 0, g_{11}(x)=0$, then there exists $g_{k l}(x) \neq 0$ satisfying $g_{(k+u) l}(x)=0$ for some $k, l$ and $1 \leq u \leq n-k$ since $G(x) \neq 0$. So $f_{11}(x) g_{k l}(x)=0$. Hence there exists $s \in R \backslash\{0\}$ such that $f_{11}(x) s=0$. Write $A=s E_{1 n}$. Then $F(x) A=A G(x)=0$.

Case 3. If $f_{11}(x)=0, g_{11}(x) \neq 0$, then there exist $A, B \in R_{n} \backslash\{0\}$ such that $F(x) A=B G(x)=0$. The proof is similar to Case 2 .

Case 4. If $f_{11}(x)=0, g_{11}(x)=0$, then for any $s \in R \backslash\{0\}, F(x) A=$ $A G(x)=0$ with $A=s E_{1 n}$.

Therefore, $R_{n}$ is linearly McCoy.

" $\Leftarrow "$. Assume that $f(x) g(x)=0$, where $f(x), g(x)$ are nonzero linearly polynomials of $R[x]$. Let $F(x)=f(x) E_{n}, G(x)=g(x) E_{n}$ with $E_{n}$ the $n \times n$ identity matrix. Then $F(x), G(x) \in R_{n}[x] \backslash\{0\}$ and $F(x) G(x)=0$. Since $R_{n}$ is linearly McCoy, there exist $A, B \in R_{n} \backslash\{0\}$ such that $F(x) A=B G(x)=0$. Obviously, there exist nonzero $a, b \in R$ such that $f(x) a=b g(x)=0$. So the proof is complete.

Given a ring $R$ and an $(R, R)$-bimodule $M$, the trivial extension of $R$ by $M$ is the $\operatorname{ring} T(R, M)=R \oplus M$ with the usual addition and the following multiplication:

$$
\left(r_{1}, m_{1}\right)\left(r_{2}, m_{2}\right)=\left(r_{1} r_{2}, r_{1} m_{2}+m_{1} r_{2}\right) .
$$

This is isomorphic to the ring of all matrices

$$
\left(\begin{array}{cc}
r & m \\
0 & r
\end{array}\right),
$$


where $r \in R$ and $m \in M$ and the usual matrix operations are used.

Corollary 3.2. A ring $R$ is linearly McCoy if and only if the trivial extension $T(R, R)$ is linearly McCoy.

However, the trivial extension $T(R, S)$ of a $\operatorname{ring} R$ by a ring $S$ being right linearly McCoy does not imply that of $S$.

Example 3.3. Let $K$ be a commutative ring, $R=\left\{\left(\begin{array}{cc}a & 0 \\ 0 & a\end{array}\right): a \in K\right\}$ and $S=$ $\left\{\left(\begin{array}{cc}a_{11} & a_{12} \\ 0 & a_{22}\end{array}\right): a_{i j} \in K\right\}$. Then the ring $R$ and the trivial extension $H=T(R, S)$ are right linearly McCoy, but $S$ is not.

Proof. Since $R \cong K, R$ is a linearly McCoy ring. But $S$ is not right linearly McCoy by [4, Proposition 10.2]. It is easy to check that $S$ is an $(R, R)$-bimodule. We next show that $H=T(R, S)$ is right linearly McCoy. Let

$$
\begin{aligned}
& F(x)=\left(\begin{array}{cc}
\left(\begin{array}{cc}
f(x) & 0 \\
0 & f(x)
\end{array}\right) & \left(\begin{array}{cc}
f_{11}(x) & f_{12}(x) \\
0 & f_{22}(x)
\end{array}\right) \\
\left(\begin{array}{cc}
0 & 0 \\
0 & 0
\end{array}\right) & \left(\begin{array}{cc}
f(x) & 0 \\
0 & f(x)
\end{array}\right)
\end{array}\right) \text { and } \\
& G(x)=\left(\begin{array}{cc}
\left(\begin{array}{cc}
g(x) & 0 \\
0 & g(x)
\end{array}\right) & \left(\begin{array}{cc}
g_{11}(x) & g_{12}(x) \\
0 & g_{22}(x)
\end{array}\right) \\
\left(\begin{array}{cc}
0 & 0 \\
0 & 0
\end{array}\right) & \left(\begin{array}{cc}
g(x) & 0 \\
0 & g(x)
\end{array}\right)
\end{array}\right)
\end{aligned}
$$

be nonzero linear polynomials of $H[x]$ with $F(x) G(x)=0$, where $f(x)=a+a^{\prime} x$, $f_{i j}(x)=a_{i j}+a_{i j}^{\prime} x, g(x)=b+b^{\prime} x, g_{i j}(x)=b_{i j}+b_{i j}^{\prime} x \in K[x]$ and $1 \leq i \leq$ $j \leq 2$. From $F(x) G(x)=0$, we have $f(x) g(x)=0, f(x) g_{11}(x)+f_{11}(x) g(x)=$ $0, f(x) g_{12}(x)+f_{12}(x) g(x)=0$ and $f(x) g_{22}(x)+f_{22}(x) g(x)=0$. For any $s \in K$, write $E_{12}(s)=\left(\begin{array}{ll}O & \left(\begin{array}{ll}s & s \\ 0 & s\end{array}\right) \\ O & O\end{array}\right) \in H$.

Case 1. If $f(x)=0$, then for any nonzero $r \in K$, let $A=E_{12}(r)$. Then $F(x) E_{12}(r)=0$.

Case 2. If $f(x) \neq 0, g(x) \neq 0$, then there exists $s \in K \backslash\{0\}$ such that $f(x) s=0$ since $f(x) g(x)=0$ and $K$ is linearly McCoy. Let $B=E_{12}(s)$. Then $F(x) B=0$.

Case 3. If $f(x) \neq 0, g(x)=0$, then $f(x) g_{11}(x)=f(x) g_{12}(x)=f(x) g_{22}(x)=$ 0 . Note that $G(x) \neq 0$. Without loss of generality, we may assume that $g_{11}(x) \neq$ 0 . So there exists $t \in K \backslash\{0\}$ such that $f(x) t=0$. Write $C=E_{12}(t)$. Then $F(x) C=0$.

Hence, $H$ is a right linearly McCoy ring.

Remark 3.4. Based on Proposition 3.1, one may suspect that the matrix ring and the upper triangular matrix ring over a linearly McCoy ring are linearly McCoy. But it gives a negative answer by [4, Proposition 10.2]. So the linearly McCoy property is badly behaved with regards to Morita invariance.

In view of [5, Example 2.1], the class of linearly McCoy rings is not closed under homomorphic images. Nevertheless, we have the following theorem. 
Theorem 3.5. Let $R$ be a ring and $n$ any positive integer. If $R$ is linearly McCoy, then $R[x] /\left(x^{n}\right)$ is a linearly McCoy ring, where $\left(x^{n}\right)$ is the ideal generated by $x^{n}$.

Proof. Denote $\bar{x}$ in $R[x] /\left(x^{n}\right)$ by $u$. Then $R[x] /\left(x^{n}\right) \cong R[u]=R+R u+\cdots+$ $R u^{n-1}$, where $u$ commutes with elements in $R$ and $u^{n}=0$.

Let $F(y)=f_{0}(u)+f_{1}(u) y, G(y)=g_{0}(u)+g_{1}(u) y$ be nonzero elements of $R[u][y]$ such that $F(y) G(y)=0$, where $f_{i}(u)=\sum_{p=0}^{n-1} a_{i}^{p} u^{p}$ and $g_{j}(u)=$ $\sum_{q=0}^{n-1} b_{j}^{q} u^{q}$ with $i, j=0,1$. Then

$$
\begin{aligned}
0 & =F(y) G(y) \\
& =\left(f_{0}(u)+f_{1}(u) y\right)\left(g_{0}(u)+g_{1}(u) y\right) \\
& =\left(\sum_{p=0}^{n-1} a_{0}^{p} u^{p}+\sum_{p=0}^{n-1} a_{1}^{p} u^{p} y\right)\left(\sum_{q=0}^{n-1} b_{0}^{q} u^{q}+\sum_{q=0}^{n-1} b_{1}^{q} u^{q} y\right) \\
& =\left[\sum_{p=0}^{n-1}\left(a_{0}^{p}+a_{1}^{p} y\right) u^{p}\right]\left[\sum_{q=0}^{n-1}\left(b_{0}^{q}+b_{1}^{q} y\right) u^{q}\right] .
\end{aligned}
$$

In particular, we have

$$
\left(a_{0}^{0}+a_{1}^{0} y\right)\left(b_{0}^{k}+b_{1}^{k} y\right)=0
$$

with minimal $k$ such that $b_{0}^{k}+b_{1}^{k} y \neq 0$. Such $k$ exists since $G(y) \neq 0$.

Assume that $a_{0}^{0}=a_{1}^{0}=0$. Let $h(u)=u^{n-1}$. Then $f_{0}(u) h(u)=f_{1}(u) h(u)=$ 0 , whence $F(y) h(u)=0$ since $u^{n}=0$.

Suppose that $a_{i}^{0} \neq 0$ for some $i$. Since $R$ is linearly McCoy, Eq. (*) implies that there exists $r \in R \backslash\{0\}$ such that $\left(a_{0}^{0}+a_{1}^{0} y\right) r=0$. Let $h(u)=r u^{n-1}$. Then $f_{i}(u) h(u)=0$ for $i=0,1$, and thus $F(y) h(u)=0$.

Hence $R[x] /\left(x^{n}\right) \cong R[u]$ is right linearly McCoy. $R[x] /\left(x^{n}\right)$ is left linearly McCoy can be shown in the same manner.

A classical right quotient ring for $R$ is a $\operatorname{ring} Q$ which contains $R$ as a subring in such a way that every regular element (i.e., non-zero-divisor) of $R$ is invertible in $Q$ and $Q=\left\{a \mu^{-1}: a, \mu \in R, \mu\right.$ regular $\}$. The free algebra $L\langle x, y\rangle$ in two indeterminates over a field $L$ is a well-known example of a domain which does not have a classical right quotient ring.

Theorem 3.6. Suppose that there exists the classical right quotient ring $Q$ of a ring $R$. Then $R$ is right linearly McCoy if and only if $Q$ is right linearly McCoy.

Proof. " $\Rightarrow$ ". Let $f(x)=\alpha_{0}+\alpha_{1} x$ and $g(x)=\beta_{0}+\beta_{1} x \in Q[x] \backslash\{0\}$ satisfy $f(x) g(x)=0$. By [13, Proposition 2.1.16], we may assume that $\alpha_{i}=$ $a_{i} u^{-1}, \beta_{j}=b_{j} v^{-1}$ with $a_{i}, b_{j} \in R$ for $i, j=0,1$ and regular elements $u, v \in R$. For each $j$, there exist $c_{j} \in R$ and a regular element $w \in R$ such 
that $u^{-1} b_{j}=c_{j} w^{-1}$ also by [13, Proposition 2.1.16]. Denote $f_{1}(x)=a_{0}+a_{1} x$ and $g_{1}(x)=c_{0}+c_{1} x$. Then the equation

$$
\begin{aligned}
f_{1}(x) g_{1}(x)(v w)^{-1} & =\sum_{i=0}^{1} \sum_{j=0}^{1}\left(a_{i} c_{j}\right)(v w)^{-1} x^{i+j} \\
& =\sum_{i=0}^{1} \sum_{j=0}^{1} a_{i}\left(u^{-1} b_{j}\right) v^{-1} x^{i+j} \\
& =f(x) g(x)=0
\end{aligned}
$$

implies $f_{1}(x) g_{1}(x)=0$. Since $R$ is right linearly McCoy, there exists $s \in R \backslash\{0\}$ such that $f_{1}(x) s=0$, i.e., $a_{i} s=0$ for $i=0,1$. Then $\alpha_{i}(u s)=a_{i} s=0$ for every $i$, which implies that $f(x)(u s)=0$ and $u s$ is nonzero in $Q$. This proves that $Q$ is right linearly McCoy.

"६". Let $f(x)=a_{0}+a_{1} x, g(x)=b_{0}+b_{1} x \in R[x] \backslash\{0\} \subseteq Q[x] \backslash\{0\}$ satisfy $f(x) g(x)=0$. Then there exists $\alpha \in Q \backslash\{0\}$ such that $f(x) \alpha=0$ since $Q$ is right linearly McCoy. Because $Q$ is a classical right quotient ring, we can take $\alpha=$ $a u^{-1}$ for some $a \in R \backslash\{0\}$ and regular element $u$. Then $f(x) a u^{-1}=f(x) \alpha=0$, implies that $f(x) a=0$. Therefore, $R$ is a right linearly McCoy ring.

Goldie theorem reveals that if $R$ is a semiprime two-sided Goldie ring, then $R$ has the classical left and right quotient rings. Hence there exists a class of rings satisfying the following hypothesis.

Corollary 3.7. Suppose that there exists the classical left and right quotient ring $Q$ of a ring $R$. Then $R$ is linearly McCoy if and only if $Q$ is linearly McCoy.

\section{References}

[1] D. D. Anderson and V. Camillo, Armendariz rings and Gaussian rings, Comm. Algebra 26 (1998), no. 7, 2265-2272.

[2] G. M. Bergman, The Diamond Lemma for ring theory, Adv. Math. 29 (1978), no. 2, $178-218$.

[3] A. M. Buhphang and M. B. Rege, Semi-commutative modules and Armendariz modules, Arab. J. Math. Sci. 8 (2002), no. 1, 53-65.

[4] V. Camillo and P. P. Nielsen, McCoy rings and zero-divisors, J. Pure Appl. Algebra 212 (2008), no. 3, 599-615.

[5] J. Cui and J. L. Chen, Linearly McCoy rings and their generalizations, Commun. Math. Res. 26 (2010), no. 2, 159-175.

[6] _ On McCoy modules, Bull. Korean Math. Soc. 48 (2011), no. 1, 23-33.

[7] Y. Hirano, On annihilator ideals of a polynomial ring over a noncommutative ring, J. Pure Appl. Algebra 168 (2002), no. 1, 45-52.

[8] C. Huh, Y. Lee, and A. Smoktunowicz, Armendariz rings and semicommutative rings, Comm. Algebra 30 (2002), no. 2, 751-761.

[9] Y. C. Jeon, H. K. Kim, Y. Lee, and J. S. Yoon, On weak Armendariz rings, Bull. Korean Math. Soc. 46 (2009), no. 1, 135-146.

[10] M. T. Koşan, Extensions of rings having McCoy condition, Canad. Math. Bull. 52 (2009), no. 2, 267-272. 
[11] T. K. Lee and T. L. Wong, On Armendariz rings, Houston J. Math. 29 (2003), no. 3, 583-593.

[12] Z. Lei, J. L. Chen, and Z. L. Ying, A question on McCoy rings, Bull. Aust. Math. Soc. 76 (2007), no. $1,137-141$

[13] J. C. McConnell and J. C. Robson, Noncommutative Noetherian Rings, Wiley, New York, 1987.

[14] N. H. McCoy, Remarks on divisors of zero, Amer. Math. Monthly 49 (1942), 286-295.

[15] P. P. Nielsen, Semi-commutativity and the McCoy condition, J. Algebra 298 (2006), no. $1,134-141$.

[16] M. B. Rege and S. Chhawchharia, Armendariz rings, Proc. Japan Acad. Ser. A Math. Sci. 73 (1997), no. 1, 14-17.

[17] Z. L. Ying, J. L. Chen, and Z. Lei, Extensions of McCoy rings, Northeast. Math. J. 24 (2008), no. $1,85-94$.

JIAN CUI

Department of Mathematics

Anhui Normal University

Wuhu 241000, P. R. China

E-mail address: jcui1635@gmail.com

JiANLONG Chen

Department of Mathematics

SOUtheast University

NANJing 210096, P. R. China

E-mail address: jlchen@seu.edu.cn 